\title{
EMI MOOCS FOR UNIVERSITY LECTURERS
}

\author{
Elena Belyaeva \\ St Petersburg State University, Russia \\ Phone: +7 (981) 890 1354, E-Mail: lena.belyaeva@gmail.com
}

\begin{abstract}
English as a Medium of Instruction (EMI) at higher education institutions (HEIs) is gradually becoming more and more important and has a unique range of issues clearly different from those of EAP and ESP. EMI in higher education can facilitate the growth of the National Higher Education export potential and help each and every HEI move up the university rankings due to the growing numbers of international students. There is clearly a need for more university courses taught in English and EMI in higher education has already been discussed extensively in a number of international scholarly journals promoting globalization and internationalization of higher education. A smallscale investigation has been made to find out what kind of professional support is currently available for university faculty teaching and lecturing in English. This investigation has been limited to massive open online course (MOOC) platforms and international teaching qualifications providers. A brief outline of the research findings with an overview of the EMI-related MOOCs targeted at university faculty is offered with a detailed comparative analysis of the following MOOCs: "English as a Medium of Instruction for Academics" developed by the University of Southampton on the FutureLearn platform and "English for Teaching Purposes" developed by the Open University of Barcelona on the Coursera platform. The comparative analysis will cover the following variables: syllabus statement, course content, methods and techniques, assessment, resources and some learning outcomes. This paper is aimed at the university faculty who are already teaching their disciplines through English or will be working in academic international contexts in English with international cohorts of students in a global classroom.
\end{abstract}

Key words: EMI (English as a Medium of Instruction); HE (Higher Education) internalization; EAP (English for Academic Purposes); instructional English; English for teaching purposes, intercultural competence; university faculty professional development.

\section{INTRODUCTION}

In the era of internalization and globalization of universities all over the world English has become the lingua franca of academia and has become a Medium of Instruction (EMI) in every higher education institution which works hard to increase the export potential of their education on a global higher education market and to become a truly international university. Clearly, university courses in a wide range of subjects require instruction through English. According to the British Council, the number of higher educational institutions which offer master's courses taught through English across Europe has dramatically risen from 4,646 in 2012 to 6,407 in 2013 (4). This trend 
has remained quite stable over the recent few years and, naturally, in this new context the quality of university courses taught through English has become paramount for institutions, students and lecturers alike. However, the university lecturers and teachers, who have to teach their subjects through English which is not their native or first language, face a number of serious challenges. Firstly, they have to switch to English as the language of instruction and classroom interaction. Secondly, they need to switch to more innovative university teaching methodology, often far more interactive than the one they had been practicing in their universities before. Thirdly, they have to teach an international cohort of students, both multilingual and multicultural, which is different from teaching a monolingual group of home students sharing the same cultural background.

The recently expanding EMI has also brought into focus the existing language policies, the way the EMI phenomenon influences L1 in Academia and English as the language of global science and research (3). The EMI phenomenon has raised a vital issue of EMI professional support to EMI teachers and these professional development opportunities for EMI teachers have not rocketed with the same speed as the dramatically growing number of university courses taught through English by non-native speakers. An overview of both linguistic and methodological EMI training of some European universities which have started to offer their EMI teachers is offered in Costa's article (1), which covers ten European countries (Belgium, Croatia, Denmark, Finland, France, Germany, Italy, the Netherlands, Spain and the United Kingdom). Costa's investigation helped to discover several common aspects of the various university programs: EMI training programs are typically developed and delivered by the Language Centers by language specialists but remained unknown whether the lecturers are native speakers or non-native speakers of English; EMI training is based primarily on language or methodological aspects; language issues are considered by EMI teachers as a clearly perceived need; finally, there remains a lot of confusion in EMI terminology

Among the ten European countries which were part of Costa's investigation there is a description of the two courses related to the UK and those are offered by the University of Oxford and the British Council. Since 2015 when Costa's article was published, the number of EMI trainings has increased and nowadays some other universities are offering EMI trainings to overseas university faculty on their websites. From the perspective of the author, EMI training offered by the UK institutions and taught by native speaker trainers belongs to a different category of EMI trainings and in many non-anglophone countries are viewed as superior as most of the other products developed by the native speaker part of the professional community.

The third suggested category of EMI training consists of massive open online courses or MOOCs and, therefore, this small scale research started as a search for the EMI MOOCs as the subject for investigation. So, the search included the MOOC platforms like Coursera, Openedu, Stepic, FutureLearn, Edx and some others. The two EMI MOOCs - "English for Teaching Purposes" and "EMI for Academics" have been found on the platforms Coursera and FutureLearn respectively. The comparative analysis of these two EMI MOOcs has come into the focus of this paper. 


\section{EMI MOOCS: GENERAL INFORMATION}

While English as a Medium of Instruction has clearly been acquiring a crucially important role in the overall process of HE internalization and globalization all over the non-anglophone world, the number of massive open online courses, clearly aimed at EMI skills training, is still surprisingly limited.

For the valid and reliable comparative analysis it has been decided to limit this investigation to the following two EMI online courses which have been identified as $100 \%$ relevant and suitable for this small-scale research:

- Certified MOOC "English for Teaching Purposes", online course and assessment. The course provider: "Coursera" platform, developed by the University of Barcelona (Universitat Autonoma de Barcelona) (https://www.coursera.org/learn/teachingenglish)

- Certified MOOC "English Medium of Instruction for Academics", online course and assessment. The course provider: "FutureLearn" platform, developed by the University of Southampton (https://www.futurelearn.com/courses/emi-academics)

It should be mentioned that Cambridge English Language Assessment Unit, with the University of Cambridge (www.cambridgeenglish.org/emi) also developed a Certificate in EMI Skills "English as a Medium of Instruction", online course and progress tests. However, this online course is offered to faculty through universities only, so, for the sake of the validity of the comparative analysis it has been decided not to bring the Cambridge EMI course into the focus of this analysis.

Massive open online courses have already become one of the most powerful tools of the lifelong learning and professional development for a number of good reasons. First of all, MOOCs allow for the flexibility in time, place and pace. Secondly, MOOCs offer secure and face-saving mode of learning which is not just important but paramount for HE faculty. Thirdly, MOOCs allow for a various degree of involvement and for the required support and scaffolding. The fact that MOOCs offer an international certificate had a different meaning depending on the local policy of the university management and/or national HE policy. However, regardless the university policy such a certificate does strengthen an individual academic portfolio anyway.

Looking at the general description of the two MOOCs under study - "English for Teaching Purposes" and "EMI for Academics" - it was found both courses share several general characteristics:

- The same course length of 4 weeks and the same suggested number of hours per week ranging from 16 to 20 hours per one week;

- Both courses offer free access to course for its duration, plus 14 days regardless when you join and including access to articles, videos, peer reviews, quizzes;

- Free option without a certificate;

- Upgrading the course ("English for Teaching Purposes" and "EMI for Academics") will guarantee an unlimited access to the course materials and a Certificate of Achievement;

- Both MOOCs' minimum entry requirements and prerequisites include: Independent English language user, CEFR Level B1+ and Subject specific English (ESP) competence. 
However, there are two characteristics which are slightly different:

- Coursera ("English for Teaching Purposes") does offer some support in Russian, while FutureLearn ("EMI for Academics") uses only English;

- While both MOOCs introduce 2 course teachers (1 male and 1 female), "English for Teaching Purposes" is delivered by the 2 non-native course leaders Silvia Solan and Jose Luis Ygoa and "EMI for Academics" is delivered by the 2 native speakers of English Rob Baird and Mary Page. Besides, "EMI for Academics" offers mini lectures by 2 invited speakers: Prof. Jenifer Jenkins and Dr Kristina Hultgren. So, the "EMI for Academics" offers substantial exposure to native speaker academic and scholarly English.

\section{COMPARING THE MOOCS' CONTENT AND SYLLABUS STATEMENTS}

"English for Teaching Purposes" is based on a functional syllabus and focuses primarily on instructional English and university classroom discourse. The rationale behind the selected approach is a move from CLIL (Content and Language Integrated Learning) to EMI (English as a Medium of Instruction). The major skill development focuses on developing lecturing style in English. Such an approach has somewhat an 'equipping' effect and at times has a slightly prescriptive and instructional tone of delivery.

"EMI for Academics" is clearly an example of a topic-based syllabus with an agenda to introduce the target audience to EMI and to help them learn about current EMI practice. There is also an openly stated objective to increase confidence in using English and to develop intercultural skills. Having completed both MOOCs and all the suggested tasks and activities I will allow myself to describe this course as having more of an 'enabling' and 'empowering' effect which is, from my perspective, more appealing to the university faculty, university culture and the drive for professional development and growth of highly intellectual workforce. This MOOC is far from being 'prescriptive' or 'instructional', as it offers a lot of awareness-raising issues and initiates a lot of meaningful professional- and self-reflection.

To make a truly valid comparative analysis of the course content it is useful and informative to compare widely-used 'can do' statements or, in other words, what both MOOCs claim course participants will be able to do upon the course completion.

Here is the list of the 'can do' statements of the "English for Teaching Purposes" MOOC:

- teach a university subject in English;

- integrate all aspects of CLIL to put together their own English-medium course on their particular subject;

- describe the characteristics of the university lecture discourse genre;

- understand and perform the basic and the main linguistic macro-functions within English teaching discourse;

- consolidate their English language proficiency at levels C1 and C2.

Here is the list of the 'can do' statements of the "EMI for Academics" MOOC:

- explore aspects of research and practice in contemporary EMI across the world;

- develop confidence in using EMI and become part of an EMI community;

- compare the different contexts of use for EMI and reflect upon how this understanding fits with our own teaching context; 
- identify challenges in facilitate effective intercultural communication and explore how these may be addressed;

- explore the role of the voice;

- identify appropriate language to facilitate effective communication in English;

- investigate how to promote successful communication in educational interactions.

Despite the fact that the syllabus statements are meaningfully similar, the 'can do' statements do not demonstrate the same degree of similarity. The 'can do' statements are quite in line with the selected approach to syllabus design. "English for Teaching Purposes" MOOC, with its emphasis on functions, highlights the ability "to perform the main linguistic macro-functions" and "to teach a university subject in English" which in my experience proved to be perfectly feasible. However, the feasibility of making a leap from the required $\mathrm{B} 1+$ language proficiency level up to $\mathrm{C} 1$ and/or even $\mathrm{C} 2$ according to CEFR is highly debatable. "English for Teaching Purposes" MOOC as any other course of English for specific purposes should not and need not make any claims about such dramatic improvements of the language proficiency. The number of academic hours required to make a move from $\mathrm{B} 2$ to $\mathrm{C} 1$ goes far beyond the period of four weeks and 80 hours of learning time. So, this claim is slightly deceiving and can develop a false expectation. The tutors of the "EMI for Academics" MOOC are being far more honest and do not encourage the course participants to believe in the "quick fix", but rather argue that the minimum required language level of English to confidently and effectively teach a university subject through English should be at least B2+, but $\mathrm{C} 1$ is the preferred language proficiency level.

"EMI for Academics" MOOC's 'can do' statements are formulated with the help of the verbs and verbal phrases like explore, develop confidence, identify challenges, explore the role of, investigate, compare, reflect upon. The implication is that all the course participants have a meaningful opportunity to succeed to a different degree depending on their motivation, time and energy invested into the MOOC, EMI experience and the current language proficiency. However, every "EMI for Academics" 'can do' statement sounds like specific, relevant and achievable.

EMI knowledge and skills have been extensively discussed (...) and generally defined as the combination of the following components:

- instructional English required for both undergraduate- and postgraduate-level university teaching;

- university classroom discourse required for managing a wide variety of classroom modes and the complex interaction with students;

- intercultural skills for effective management of multicultural and multilingual classrooms;

- higher education modes of lecturing and teaching.

If we take a closer look at the content of the investigated MOOCs to identify which components have come into their focus, we will have to state that "English for Teaching Purposes" is fully focused on the instructional English and classroom discourse. On the other hand, all the four weeks' content consistently reveals the functional approach, where the first week's is devoted to implications for lecturers switching to English as the classroom language at university, and teacher discourse; the second week focuses on the main sequences of teacher discourse: explaining, justifying and all the functions related to clarifying concepts for the students; the third week is devoted to functions related to information management, 
evaluation, induction and socialization; the fourth week is fully dedicated to peer review and feedback of the recorded classes and/or session fragments. So, "English for Teaching Purposes" does not include any information and/or training aimed at the development of university teaching methodology or intercultural skills and competencies.

"EMI for Academics" MOOC with its topic-based syllabus covers a wider range of the EMI components including deep discussions on what EMI actually is with different contexts and interpretations of the term. During the first week the course also introduces course participants into the latest research findings and discussions about the influence of EFL research on EMI. The second week's materials are a blend of the useful language for presenting ideas in English (signposting language), the discussion of how far accuracy is important in an EMI classroom and the role of the language in intercultural communication. The fourth week is devoted to the issues and challenges in lecturing for a multilingual and multicultural audience, avoiding stereotypes and recognizing cultural differences. The fourth week is all about facilitating and managing effective communication in small groups and seminars and these are clearly university teaching methodology aspects.

Obviously, "EMI for Academics" covers the full range of components of the EMI knowledge and skills, therefore more than "English for Teaching Purposes". On the one hand, one may argue that "English for Teaching Purposes" MOOC has a stronger chance for a considerable improvement of instructional English and classroom discourse. But on the other hand, there are missed opportunities in terms of intercultural competence, development together with a number of vitally important and highly useful discussions of a number of issues like using other languages in the classroom or translanguaging, English as a global language, pronunciation versus intelligibility, etc.

\subsection{Comparing the language components in the investigated MOOCs}

The language content is described and specified in terms of language functions, however, "English for Teaching Purposes" offers a comprehensive list of the following instructional language functions: explaining and justifying, beginning and ending a class, classifying and categorizing, comparing and contrasting, giving examples, defining, describing processes and visuals, expressing cause and effect, expressing certainty, presenting evidence, giving opinions.

"EMI for Academics" also has got certain general language functions: signposting language, language to structure your lecture, managing the classroom conversation, etc. However, it has a number of language functions which feel a lot more course participant centered: being persuasive, making an emphasis, telling anecdotes, etc. This course has got an agenda to help course participants improve their lecturing styles, develop their teaching identity through English, not to "regress" too much in terms of their personality and communicative culture which should find their way to the audience through English. Along with all the above mentioned there is also an extensive discussion of the changing norms of English as a global language. Special attention is given to the role of pronunciation in the EMI classroom, and intelligibility is suggested as the major focus for improvement.

The language component is obviously treated in a different way and at a different level. Clearly the range of language functions to be developed through "English for Teaching Purposes" are the core ones for the EMI context helping course participants to shape up the foundation of the instructional English and classroom discourse. "EMI for Academics" MOOC exercises a slightly more customized and client-oriented approach to 
offering various language development opportunities to highly intellectual, well-informed and well-educated people (which most of the university faculty staff typically are).

Language component is the core component in the "English for Teaching Purposes" MOOC, just as in the "EMI for Academics" MOOC. However, having done both MOOCs experientially, and having analyzed the content of the language work in both MOOCs, we argue that all those potential course participants, who are currently at B1+ and B2 level, should be recommended to start with the "English for Teaching Purposes" and then to move on towards the "EMI for Academics" when they can make a breakthrough towards C1 and above. Those potential course participants who have already acquired $\mathrm{C} 1+$ level of English are recommended to take "EMI for Academics" straight away until and unless they have got a particular language problem with one or several language functions. If this is the case, then "English for Teaching Purposes" might be of use and help to them with a specific language puzzle.

\section{COMPARING TEACHING AND LEARNING TECHNIQUES}

In the previous sections of the paper the 'what' component was central to the comparative analysis and the focus was on the course content of the investigated MOOCs. Yet another important variable to explore is the 'how' component which means the collection of the selected teaching techniques which together form an approach.

During the past decades, we have observing the online education boom with a vast number of MOOCs rocketing on various open online platforms. However, despite the widest variety in course content, the range of teaching techniques that have been most extensively used has remained quite limited. Some will argue that the online mode of learning and teaching is trapped in a certain limited range of techniques available by definition and one cannot help agreeing to that.

We are ready to agree with that when it comes to the 'input' kind of activities and if we look at the investigated MOOCs - "English for Teaching Purposes" and "EMI for Academics", we will find an almost identical list of activities meant for providing the learning input to the course participants: video talks and video transcripts available, references and links to the suggested resources. Besides, "EMI for Academics" also offers articles and academic papers and referenced texts for extended reading. So, in terms of ways of providing input, both MOOCs use basically the same repertoire of techniques. As for the amount of input, "EMI for Academics" offers a lot more of a varied input with a far stronger chance to intellectually satisfy those course participants who have the biggest 'appetite' for learning and processing a lot of input.

What most MOOCs designers try to do is to make them interactive, so that there is an exchange of opinions and ideas, course leader - course participants interaction, course participants interaction, feedback, question - answer opportunities, reflection and selfreflection, cooperative activities and competitive activities as well. This is not input, but more of output nature activities supposed to enthuse course participants to respond, interact, participate, react and share, so that the course is gradually becoming more of a 'two-way street' with a growing number of course participants being truly involved.

Let us examine the kind of interactive activities the investigated MOOCs employ. Both courses have forums. However, those forums are different in terms of the numbers of people involved, the discussion dynamics and the intensity of interaction. "English for 
Teaching Purposes" demonstrates what can be defined a formal approach to forum management with minimal monitoring and facilitation efforts on the course leaders' part. "EMI for Academics" has got a very intellectually-stimulating and thought-provoking forum with relevant and thoughtful contributions. This is a convincing example of a forum being a truly learning activity where course participants are actively learning from each other. This is difficult to overestimate as the course leaders should not be the only source of knowledge and information, particularly when it comes to an international cohort of course participants where every voice is contributing to an overall panorama of EMI all around the world allowing for useful comparisons to be made and various reflections to be triggered.

Discussing forum as an extensively exploited online course activity, it should be mentioned that apparently the course leaders' attitude and involvement are paramount in shaping the course participants' attitudes and raising the level of involvement. "EMI for Academics" course leaders - Rob Baird and Mary Page - do take an active part in monitoring all the discussion forums. There is no doubt that they both read all the postings, quite often they respond with some very relevant posting to an individual comment. Sometimes they step in when the discussion seems to be stuck and they provide helpful insight which indicates a new perspective or a different direction of the group discussion. Once in a while both course leaders 'appear' on the forum through U-tube channel to summarize the discussion on the topic-based forum and what appeals to the course participants is that they refer to certain course participants' comments, they address those course participants by their names and express openly which comments they agree with, which comments they find controversial and why, and thank course participants for their contributions again being very specific about the ideas. Often the two teachers respond to different comments and, as a result, no meaningful forum posting is left unattended, which lifts the overall level of scholarly argument and strengthens the feeling of a true dialogue and a continuous discussion and debate.

"English for Teaching Purposes" course leaders - Silvia Solan and Jose Ygoa - do not make any postings on the course forum and, possibly, according to their approach, forum should be the course participants' space where they feel secure and free to discuss things without any inhibitions. It is quite possible that the MOOC leaders have been moving on the assumption that forum should be the 'teacherless' zone and if this is the case, then we assume that this is the course approach. However, within the 4 weeks of this MOOC, an impression of this forum being 'one-way street' is likely to be developed.

Another impressive example of an interactive activity is an opinion poll in a form of a questionnaire, where all the course participants respond to the questions and then see the overall results and how they are changing depending on every single response. This is a very good idea, its professional design stimulates participation and makes everyone curious about the outcomes. The "EMI for Academics" team is getting valuable data which can be effectively used throughout the course in the future and become convincing evidence in a scholarly argument.

It should also be emphasized that "EMI for Academics" teaching team use task-based approach a lot. Even if we compare the wording of the teaching techniques described we will notice the following difference: "English for Teaching Purposes" has got "reflection and self-reflection", while "EMI for Academics" has got "reflective and self-reflective tasks". And we would argue that it does make a difference. A completed task is a far more convincing evidence of the reflection or self-reflection taking place and showing 
itself in action. "EMI for Academics" also has got what they call "scenario-based tasks" which is a detailed description of a fairly typical challenging situation of an EMI classroom and all the course participants are supposed to be sharing how they would address this kind of issue and many participants often suggest more than one way but rather a strategy what they would do first and what they would switch to next in case the first option does not bring the desirable effect or does not really help the situation. There is also an opportunity to discuss the suggested tactics, argue, generalize, expand, add and modify. This is a real learning situation in response to a plausible scenario which some participants easily recognize. In this case the meaningful experience exchange begins. It can also be categorized in a way as some kind of 'what if' planning. It all depends on how experienced course participants are in teaching multilingual and multicultural students. Needless to say, the less experienced EMI teachers are learning a lot from those who have been teaching through English for a while and have experientially discovered ways of dealing with certain typical EMI puzzles and challenges.

Quizzes which are offered to the "EMI for Academics" are a very good example of a competitive activity or a task. Unlike tests which are part of the "English for Teaching Purposes", quizzes can be classified as teaching activities because they allow going back to the same questions over and over again. Besides, a very detailed feedback is offered in case your answer to the quiz question is incorrect. This feedback to each and every question shows unprecedented dedication of the teaching team and helps a lot to see where and why your way of thinking goes wrong. Such an approach to giving feedback rather than just a score is a lot more developmental and helpful. It also has an inspiring and empowering effect. A university teacher needs a bit of a convincing and truly scholarly argumentation in order to accept and agree with a different or an opposing view or opinion. So, "EMI for Academics" has very strong chances to influence course participants in such a way that they become willing to listen and hear the arguments and, as a result, get ready to question (if not to change) their own views and positions. Besides, the vast material covered by the quizzes does have a strong motivating and inspiring effect as the questions in quizzes sometimes cover some marginal issues in the recommended reading, not just the core and key course concepts.

To sum it up, it should be stated that "EMI for Academics" demonstrates a wider range and variety of teaching activities and techniques than that offered by "English for Teaching Purposes". The exercises designed by the "EMI for Academics" teaching team approach to educating the university faculty do seem to be working better with the highly educated faculty. The reason behind that is that scholars respond better to activities of a more sophisticated nature in a professional exchange mode, rather than getting a list of things to do or just prescriptive 'gimmicks'. "EMI for Academics" is designed in such a professional way that within every week a variety of tasks is offered and this helps to escape the feeling of routine, boredom and repetition.

"English for Teaching Purposes" demonstrates a different approach which, firstly, narrows the amount of content to be covered to primarily instructional English and classroom discourse and, secondly, through repetitive tasks and activities, has a stronger chance to achieve most of the course aims and objectives. 


\section{DEALING WITH EMI TEACHERS' CONCERNS}

The potential risks, pitfalls and concerns of the target readership for this paper and the target beneficiary for this investigation - the higher education faculty members teaching their subjects and disciplines through English, which is not their native language, and working with international cohorts of students - have been extensively discussed. Below is the list of the most widely debated concerns:

- subject content is simplified due to a limited general language proficiency and/or subject-specific English;

- classroom activities and modes limited repertoire due to the existing limitations in instructional English and interactive classroom discourse;

- a disturbing degree of an expected 'regress' when switching from one's native language to English;

- risk of loss of face;

- teacher personality and teaching style finding its way through the medium of English;

- multicultural classroom: dealing with tensions and unease;

- slow down in teaching pace leading to the risk of the syllabus being not fully covered;

- inability to adjust the level of language complexity while dealing with mixed language proficiency groups of students;

- unimpressive learning outcomes;

- reduced job satisfaction and/or lack of sense of achievement.

This is just a brief summary of all the most frequently articulated concerns and fears of EMI teachers in order to investigate if the EMI MOOCs make an attempt to address and deal with the above mentioned EMI teachers' professional concerns.

"English for Teaching Purposes" deals with anxiety which might be an issue for those EMI teachers who have no or very little experience of teaching their specific disciplines in English. There is also a rather helpful discussion of how to control one's nervousness which is often rooted in the insecurity of teaching through English, particularly those teacher roles which require giving instructions, motivating students, managing student questions, managing classroom interaction, dealing with intercultural comments and talking spontaneously. Among the suggested ways there is peer observation, selfreflection and reflection, making the best use of the students at the advanced level of language proficiency and resourcing them in classroom.

"EMI for Academics" is clearly aimed at confidence building as it gives meaningful time to discovering ways how to create positive classroom climate and how to promote successful communication in educational interactions. This MOOC creates multiple opportunities for sharing and caring and building a professional support system for EMI teachers encouraging all the course participants to join an international community of EMI teachers.

If we are to compare and contrast the investigated MOOCs, it should be mentioned that "English for Teaching Purposes" makes course participants feel as students, while "EMI for Academics" fosters more of a 'colleague' feeling. Through this investigation it has been discovered that no MOOC can possibly cover fully the above given list of EMI teachers' concerns, but both MOOCs do make an attempt to deal with those despite all the limitations of online courses. 


\section{ASSESSMENT TECHNIQUES}

The assessment techniques often are almost identical to the teaching techniques and MOOCs are no exception. It is more interesting to explore the weight given to continuous or final assessment and here a dramatic difference has been discovered: "English for Teaching Purposes" gives a lot more weight to the final assessment which takes the form of a mini lecture or a fragment of a lecture (of approximately 12-15 minutes duration) to be filmed and then sent for peer review. One is only allowed to view other course participants' videos and to carry out a peer review or evaluation after one has downloaded his or her own video and this is certainly fair.

Needless to say, it is a bit stressful because it has to be a real fragment of a real lecture or a session with real students. It can also include an interactive communication with students, e.g. dealing with their questions. This is believed to be a valid form of assessment as an authentic sample of a professional activity demonstrating a wide range of professional competencies including pedagogic, subject-specific, presentation skills and the English language proficiency. The guidelines for peer observation and review are given and explained, so the whole process is being monitored and there is quality assurance and control by the course leaders' team.

The final assessment is also quite high stakes because without it one cannot get a Certificate of Achievement. Obviously, it is not feasible to squeeze all that is covered through the MOOC language functions within the given time limit, so there is a danger that the testtaker will have the opportunity to demonstrate what he/she can do best and up to the standard. A 15-minute slot can only offer a glimpse of the lecturing style, the language proficiency, presentation skills and classroom management competencies. However, this is a direct testing technique when people do what they would do in real life. So, the validity is definitely there to a certain degree. Reliability is a more serious issue here but the MOOC teaching team is moving on the assumption that course participants are motivated to benefit from this training and to make the most of it for their professional development and growth. What every course participant gets is peer review feedback with positive and constructive comments which is supposed to stimulate further professional growth and development.

"EMI for Academics" gives more weight to continuous assessment with basically nothing that looks like the final test or a final assignment. So, every course participant is assessed on a continuous basis and the system encourages everyone to go through the course materials step by step showing progress in percentage for every unit. It gives a clear picture where everyone is and allows for influencing the situation according to one's ambitions and plans. The Certificate of Achievement shows an overall score that is the sum of all the tests, quizzes and tasks together with the percentage of the successfully fulfilled tasks and assignments for every course unit.

So, the investigated MOOCs demonstrate different approaches to assessment and therefore, there is an opportunity to choose the preferred mode of assessment when making a decision which EMI MOOC to take. 


\section{CONCLUSIONS}

As a result of this small-scale investigation of the two selected EMI MOOCs there is evidence which suggests that both EMI MOOCS have strong chances to enable the target audience - university faculty teaching staff:

- to make a move towards a wider variety of interactive teaching techniques and use interactional English speech more confidently;

- to develop academic presentation skills in English and use transactional English speech accurately and fluently;

- to become more sensitive to intercultural communication issues;

- to develop sufficient university classroom discourse to manage interactive learning;

- to become part of a bigger EMI professional community and to network internationally for ideas and experience exchange;

- to raise their awareness of the English as a global language with dynamically changing norms and standards.

However, there are a couple of EMI teachers' challenges that have not yet been addressed by the investigated EMI MOOCs:

- how to become an efficient autonomous language learner;

- how to become an expert English language user;

- how to become an independent language problem solver.

Having done both EMI MOOCs for the purposes of the described research and in the light of the hands-on experience and language teaching expertise, the following recommendations are offered to EMI teachers who are seeking online opportunities for their professional development:

- "English for Teaching Purposes" is a very good introduction into EMI and an extremely useful induction for those EMI teachers who:

- Have no or limited EMI teaching experience;

- Have B1+ CEFR level of English;

- Need to be certified as an EMI teacher.

- "EMI for Academics" is a well-designed professional development course for those EMI teachers who:

- Have some EMI teaching experience;

- Have B2+CEFR level of English;

- Are motivated to invest a lot more time than it is suggested in the course description;

- Are seeking opportunities to network with the EMI teachers and are generally curious about EMI practice outside their educational context.

Having completed both investigated EMI courses, I found it quite a rewarding experience and confidently recommend both MOOCs as an extremely convenient way of learning with a lot of flexibility in time, pace, place, very secure and non-threatening mode, with a lot of scaffolding and support and, finally, with an international certification. EMI MOOCs have a lot to offer to non-native EMI teachers in terms of linguistic and methodological aspects. Confidence building and developing one's individual lecturing style might also be important for EMI teachers. EMI MOOCs, each to a different degree, facilitate global professional networking and are making a meaningful contribution to the international EMI teachers professional community. 


\section{REFERENCES}

Costa, F. (2015) 'English Medium Instruction (EMI) Teacher Training Courses in Europe'. Ricognizioni, Vol. 2, No 4, pp. 127-135

Marsh, D. (2006) 'English as a medium of instruction in the global linguistic order: Global characteristics, Local Consequences'. Proceedings of the Annual Conference for METMaC, 2: pp. 29-38

Mauranen, A., Hynninen, N., Ranta, E. (2010) English as an Academic Lingua Franca: the ELFA Project, in "English for Specific Purposes", 29: 183-190

The British Council booklet, 2013 Academic Teaching Excellence. Academic Teaching Skills - English as a Medium of Instruction.

Tange, H. (2010) Caught in the Tower of Babel: University lecturer's experiences with internationalization. Language and Intercultural Communication, 10(2), pp.137-149 